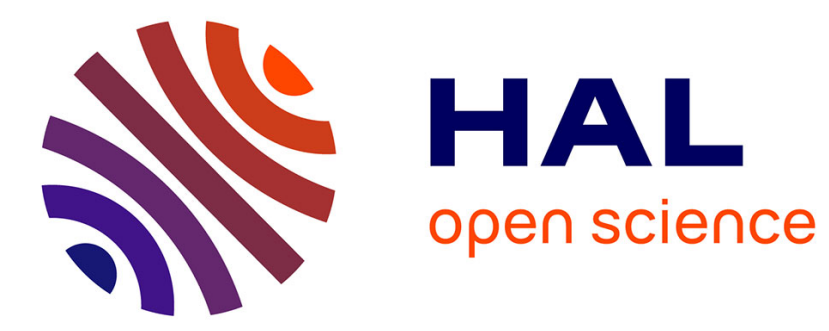

\title{
ANALYSE DES SURFACES SOLIDES PAR S.I.M.S. UTILISANT UN FAISCEAU PRIMAIRE DE PARTICULES NEUTRALISÉES
}

G. Borchardt, Sébastien J. Weber, H. Scherrer, S. Scherrer

\section{- To cite this version:}

G. Borchardt, Sébastien J. Weber, H. Scherrer, S. Scherrer. ANALYSE DES SURFACES SOLIDES PAR S.I.M.S. UTILISANT UN FAISCEAU PRIMAIRE DE PARTICULES NEUTRALISÉES. Journal de Physique Colloques, 1984, 45 (C2), pp.C2-801-C2-804. 10.1051/jphyscol:19842184 . jpa00223859

HAL Id: jpa-00223859

https://hal.science/jpa-00223859

Submitted on 1 Jan 1984

HAL is a multi-disciplinary open access archive for the deposit and dissemination of scientific research documents, whether they are published or not. The documents may come from teaching and research institutions in France or abroad, or from public or private research centers.
L'archive ouverte pluridisciplinaire HAL, est destinée au dépôt et à la diffusion de documents scientifiques de niveau recherche, publiés ou non, émanant des établissements d'enseignement et de recherche français ou étrangers, des laboratoires publics ou privés. 


\title{
ANALYSE DES SURFACES SOLIDES PAR S,I,M,S, UTILISANT UN FAISCEAU PRIMAIRE DE PARTICULES NEUTRALISÉES
}

\author{
G. Borchardt, S. Weber, H. Scherrer et S. Scherrer \\ Laboratoire de Physique du Solide (L.A. 155), E.N.S.M.I.M., Pare de Saurupt, \\ 54042 Nancy Cedex, France
}

Résumé - La méthode S. I. M. S. utilisant des particules primaires neutraIisées (NPB-SIMS) appliquée aux isolants permet d'analyser les ions secondaires positifs ou négatifs sans modifier la distribution des éléments mobiles dans le solide. Les conditions à respecter concemant les champs électriques induits et les mobilités des éléments sont discutées poun des matériaux tels que les oxydes et verres silicatés.

\begin{abstract}
Normally the analysis of insulators by the S. I. M. S. method is disturbed by charge effects. The use of neutral primary particles (NPBSIMS) appreciably reduces these effects and makes possible the analysis of both positive and negative secondary ions without changing the distribution of mobile species in the solid to be analysed if certain conditions are respected. These conditions concerming internal fields and particle mobilities are discussed. Typical results are given.
\end{abstract}

\begin{abstract}
L'analyse des isolants par S. I. M. S. est perturbée par des "effets de charges". Les variations du potentiel électrostatique de la surface bombardée conduisent à des fluctuations des signaux secondaires et induisent la migration d'éléments mobiles dans le solide. De nombreux efforts ont été faits dans différents laboratoires pour réduire cet effet de change $[1,2,3,4]$.
\end{abstract}

Poun nos travaux sur la diffusion dans les silicates et l'étude des réactions chimiques à la surface des verres, nous avions besoin d'une méthode S. I. M. S. assurant des conditions d'extraction stables avec ur effet de change stationaire très faible, une résolution locale suffisante et, si possible, des vitesses de pulvérisation convenables $(\geq 0,1 \mathrm{~nm} / \mathrm{s})$. Nous avons développé à partir d'une technique déjà citée [5] une méthode d'analyse utilisant des particules primaires neutralisées (NPB-SIMS) $[6,7,8]$.

I'appareillage CAMECA SMI 300 adapté permet d'obtenir des résultats expénimentaux repnoductibles et cette méthode est devenue un procédé de routine au cours de nos études sur les verres $[9,10$ 11].

Nous rappelons succinctement ici le principe de la méthode NPB-SMMS en discutant les conditions d'application pour l'analyse des oxydes et verres silicatés.

\section{I. - PRINCIPE DE LA METHODE NPB-SIMS}

Quand un isolant est bombardé par un faisceau de particules chargées (ions) le courant résiduel $I_{r}$ en régime permanent est la somme de plusieurs contributions provenant du courant ionique primaire $I_{P}$ et des différentes émissions ioniques et électroniques secondaires [8]. Il en résulte un potentiel résiduel sur la surface bombardée : $\Delta u(\infty)=I_{\Upsilon} \cdot \operatorname{Reff}$.

Parmi les différents moyens pour mininiser $\Delta u(\infty)$, il est possible d'utiliser un faisceau primaire de bombardement constitué par des particules neutralisées. 
Ce faisceau peut être obtenu principalement par échange de charge en phase gazeuse ou par neutralisation sur des surfaces solides surtout métaliiques. Avec le microanalyseun CAMECA SMI 300, c'est le premier mécanisme qui s'est avéré le plus efficace et le dispositif utilisé a été publié par ailleurs [7]. Notons simplement qu'il est nécessaire de placer sur la surface de l'échantillon un masque métallique muni d'un trou pour permettre le bombardement à partir du faisceau primaire neutralisé et complètement défocalisé. A partin des cratères obtenus par bombardement d'une plaquette de silicium avec des particules Art et Ar\%, le rendement de neutralisation est estimé à $25 \%$ environ.

La borne stabilité dans le temps, l'uniformité de la densité du faisceau neutralisé permettent d'obtenir une résolution en profondeur suffisante pour des vitesses de pulvérisation de l'ordre de $0,3 \mathrm{~nm} / \mathrm{sec}$.

\section{II. - APPLICATION DE LA METHODE ET DISCUSSION II - 1 - Analyse d'ions secondaires positifs}

Lors de I'analyse des ions secondaires positifs une contribution importante au courant résiduel $I_{r}$ est due aux électrons émis par l'électrode d'extraction sous I'inpact des ions secondaires et attirés par l'échantillon. Dans le cas des verres silicatés, il en résulte l'apparition sur la sumface d'un potentiel additionnel $\Delta u(\infty)$ compris entre -25 et -45 volts pour une tension d'extraction de $+4,5 \mathrm{kV}$.

L'influence de ce potentiel résiduel sur la distribution en énergie des ions secondaires émis peut être conpensée par une tension auxiliaire superposée à la tension d'extraction ou par filtrage [5].

L'influence sur la distribution locale des éléments mobiles dans le solide à analyser peut être estimée à paxtir d'une comparaison élémentaire entre le flux de particules pulvérisées et le flux de diffusion d'ions monovalents du au champ électrique induit [8]. Ceci conduit à la condition suivante valable à la température ambiante :

$$
E D_{i} \gtrsim 10^{-10}\left(\mathrm{~V} \mathrm{~cm} \mathrm{~s}^{-1}\right)
$$

$E$ champ électrique $(\mathrm{V} / \mathrm{cm})$ perpendiculaire à la surface $D_{i}$ coefficient de diffusion en volume de l'élément $i\left(\mathrm{~cm}^{2} / \mathrm{s}\right)$.

Avec des valeurs expérimentales de $D$ à $340 \mathrm{~K}$ pour le sodium et l'hydrogène dans des verres type $\mathrm{Na}_{2} \mathrm{O}-\mathrm{Ca} \mathrm{O}-\mathrm{Si} \mathrm{O}_{2}\left(\mathrm{D}_{\mathrm{i}} \cong 10^{-16} \ldots 10^{-14} \mathrm{~cm}^{2} / \mathrm{s}\right)$ la condition précédente prévoit une migration possible si $\mathrm{E} i 10^{4} \mathrm{~V} / \mathrm{cm}$. Ceci $\mathrm{n}^{\prime}$ est surement pas le cas avec les valeurs de $\Delta \mathrm{u}(\infty)$ mentionnées précédemment.

\section{II - 2 - Analyse des ions secondaires négatifs}

Si l'on considère l'extraction d'ions secondaires négatifs, c'est l'émission électronique à partir de la cible qui apporte une contribution importante au courant résiduel. Par ailleurs la surface bombardée chargée positivement par rapport au porteéchantilion et au masque peut capter une certaine fraction des électrons secondaires (surtout ceux émis à partir du masque). Dans ces conditions, le courant résiduel peut s'écrire [8] :

$$
I_{r} \simeq\left|I_{p}\right|\left[\gamma_{e f f}^{T}-\gamma^{H} f^{H}\right]
$$

Yeff coefficient d'émission électronique à partir de la surface bombardée

$r^{H}$ coefficient d'émission électronique du masque

$f^{\mathrm{H}}$ nombre positif dépendant de la géométrie du système de collection et des conditions d'extraction.

Une compensation de charge nécessite que $\gamma_{\text {eff }}^{\mathrm{T}}=\gamma^{\mathrm{H}} \mathrm{f}^{\mathrm{H}}$. Dans le cas de nos conditions expérimentales, une valeur limite inférieure est estimée à $\gamma^{\mathrm{H}} \mathrm{f}^{\mathrm{H}}=0,25$. Pour les oxydes ou verres silicatés, reffe est voisin de 2 , on doit donc s'attendre à un effet de charge important. Pour paliien cette difficulté on peut évaporer une fine 
couche métallique ( $\mathrm{Au}, \mathrm{Al}, \lesssim 50 \mathrm{~nm}$ ) sur la surface de l'échantillon en plus du masque. Dans ces conditions le potentiel résiduel mesuré est égal à $\Delta u(\infty)=+30 \mathrm{~V}$. Notons que la condition (1) du paragraphe précédent est toujouns valable et que la migration d'éléments mobiles dans le solide est peu probable.

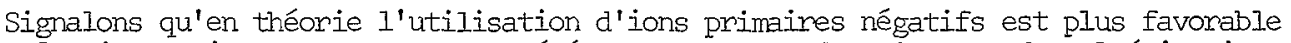
[1] mais la mise en oeuvre du procédé ne permet pas des vitesses de pulvérisation aussi grandes que la méthode NPB-SIMS.

\section{II - 3 - Exemple : corrosion d'un verre biologique dans une solution organique}

Des échantillons de verre silicaté contenant du phosphore $\left(\mathrm{Si} \mathrm{O}_{2}: \mathrm{Ca} 0\right.$ : $\mathrm{Na}_{2} \mathrm{O}$ : $\mathrm{P}_{2} \mathrm{O}_{5}=45: 24,5: 20,5: 10 \%$ en poids foumis par Saint-Gobain) ont été inmergés dans une solution de glycine $\left(\mathrm{H}_{2} \mathrm{~N}-\mathrm{CH}_{2}-\mathrm{CO}_{2} \mathrm{H}\right)$ plus $\mathrm{HCl}$ ( $\mathrm{pH}=7,4=c t e$ ), maintenue à $40^{\circ} \mathrm{C}$ pendant des temps allant jusqu'à plusieurs heures. La surface réactive est une cassure fraiche de $2 \mathrm{~cm}^{2}$ environ. La figure 1 indique quelques faits typiques de cette réaction.
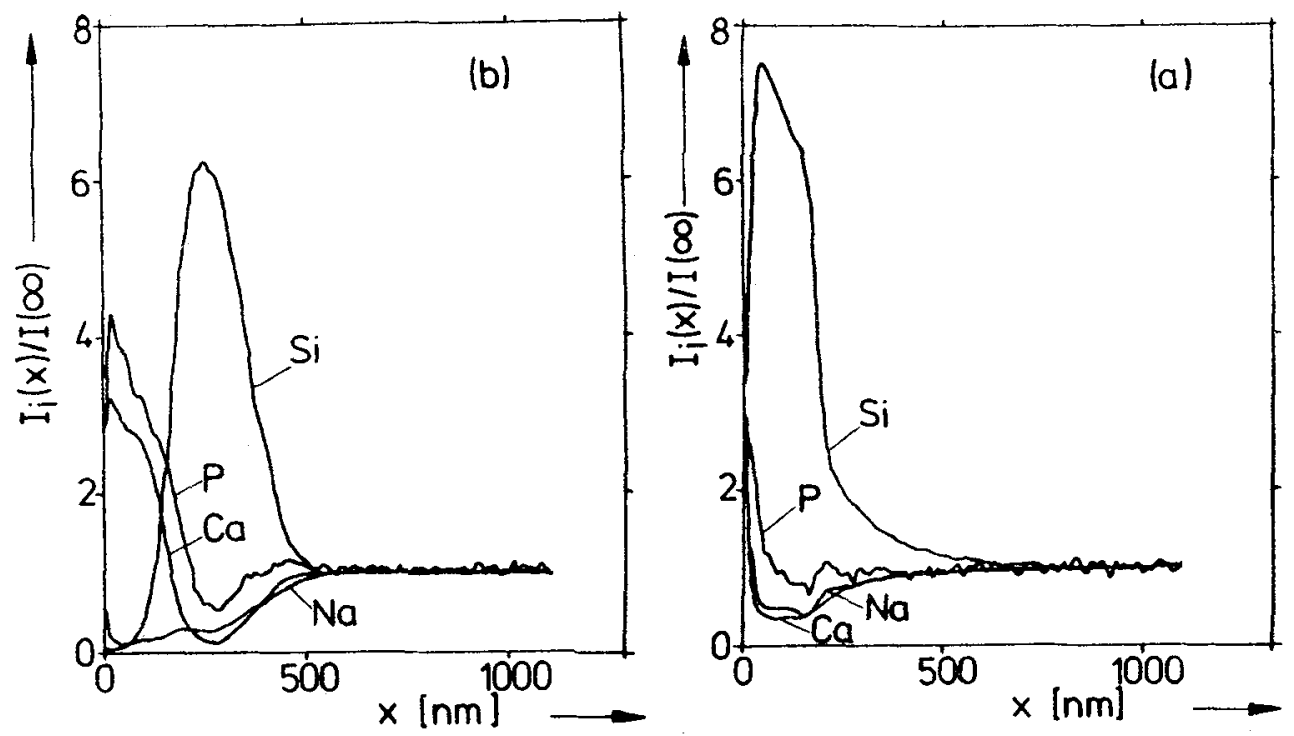

Fig. 1 - Verre biologique : profils d'intensité des éléments après corrosion pendant (a) $0,5 \mathrm{~h} ;$ (b) $1,5 \mathrm{~h}$

On y observe la formation d'une couche de verre hydraté et le développement avec le temps de couches superficielles plus riches en phosphore et calcium. Des détails expérimentaux et l'interprétation des résultats seront donnés par ailleurs [12].

\section{Réfërences.}

[1] WERNER H. W., MORGAN A. E., J. Appl. Phys., 47 (1976) 1232.

[2] GOSSINK R. G., Glass Technology, 21 (1980) 125.

[3] SLODZIAN G., HAVETTE A., J. de Microscopie et Spectroscopie Electronique, 2 (1977) 81.

[4] SLODZIAN G., DENNEBOUY E., HAVETIE A., Proc. XVIII Coll. Spectr. Int., 2 (1975) 590.

[5] BLANCHARD B., Analusis 4 (1976) 180.

[6] SCHERRER S., NAUDIN F., Proc. XI Int. Glass Congress, Prague, 3 (1977) 301. 
[7] BORCHARDT G., SCHERRER H., WEBER S., SCHERRER S., Int. J. Mass. Spectrom. Ion. Phys., 34 (1980) 361.

[8] BORCHARDT G., SCHERRER S., WEBER S., Mikrochimica Acta, Wien, II (1981) 421.

[9] THOMASSIN J. H. et al., Bull. Minéral., 102 (1979) 319.

[10] TOURAY J. C. et al., J. Non-Cryst. Solids, 38-39 (1980) 643.

[11] FRANEK H. J., Exchange Reactions between Aqueous Solutions and Glass Surfaces, Thesis, Techn. Universität Clausthal (1980)

[12] SCHERRER S. et al., J. Non-Cryst. Solids (à parâtre). 